Associate Professor Dragisa STANUJKIC, PhD

E-mail: dstanujkic@ tfbor.bg.ac.rs

Technical Faculty in Bor, University of Belgrade, Bor, Serbia

Associate Professor Darjan KARABASEVIC, PhD

E-mail: darjan.karabasevic@mef.edu.rs

Associate Professor Gabrijela POPOVIC, PhD

E-mail: gabrijela.popovic@mef.edu.rs

Faculty of Applied Management, Economics and Finance

University Business Academy in Novi Sad, Belgrade, Serbia

Professor Florentin SMARANDACHE, PhD

E-mail: smarand@unm.edu

Department of Mathematics, University of New Mexico, Gallup, USA

Professor Edmundas Kazimieras ZAVADSKAS, PhD

E-mail: Edmundas.Zavadskas@vgtu.It

Institute of Sustainable Construction, Vilnius Gediminas Technical

University, Vilnius, Lithuania

Associate Professor Ieva MEIDUTE்-KAVALIAUSKIENĖ, PhD

E-mail: ieva.meidutekavaliauskiene@vgtu.It

Business Management Faculty, Vilnius Gediminas Technical

University, Vilnius, Lithuania

\title{
MULTIPLE-CRITERIA DECISION-MAKING BASED ON THE USE OF SINGLE-VALUED NEUTROSOPHIC SETS AND SIMILARITY MEASURES
}

Abstract. As the generalization of the fuzzy and similar sets based on Fuzzy sets, neutrosophic sets provide significant possibilities in the case of solving complex decision problems, often related to uncertainty and unreliability. Neutrosophic sets use three values named the truth degree, the indeterminacy degree and the falsity degree, which allow for a more accurate evaluation of alternatives in relation to complex evaluation criteria. As a result of their application in solving numerous different decision-making problems, several approaches to their ranking have been proposed. Therefore, this paper provides a comprehensive overview of the approaches to the ranking of single-valued neutrosophic numbers and a comparison of the results obtained by using them. Finally, numerical illustrations are given.

Keywords: neutrosophy, single-valued neutrosophic set, similarity measures, MCDM.

JEL Classification: C02, C44, D80

DOI: 10.24818/18423264/55.2.21.01 
Dragisa Stanujkic, Darjan Karabasevic, Gabrijela Popovic, Florentin Smarandache, Edmundas Kazimieras Zavadskas, Ieva Meidutè-Kavaliauskienė

\section{Introduction}

In recent decades, multi-criteria decision-making (MCDM) methods have strongly been developed and have become increasingly popular (Ulutaş et al., 2020). Due to such strong development, a large number of multi-criteria decisionmakings techniques have been introduced by scholars throughout the world (Stanujkic et al., 2019; 2017). So far, MCDM has successfully been applied in solving numerous decision-making problems in different areas. Significant progress in MCDM was made after Zadeh (1965) introduced fuzzy set theory, thus providing an efficient approach to solving a much larger number of real-world decision-making problems. An important characteristic of fuzzy sets is that they use the membership function to represent the degree of belonging to a set. Different shapes of membership functions are proposed, such as triangular, trapezoidal and bell-shaped so as to enable the efficient solving of different decision-making problems.

In order to enable solving more complex problems, some extensions of fuzzy set theory have been proposed, such as interval-valued fuzzy sets (Turksen, 1986), intuitionistic fuzzy sets (Atanassov, 1986), interval-valued intuitionistic fuzzy sets (Atanassov \& Gargov, 1989), the bipolar fuzzy set (Lee, 2000), Pythagorean fuzzy sets (Yager, 2013) and so on.

In addition to more complex membership functions, these extensions also bring new mathematical operations on sets, as well as new membership functions. In bipolar fuzzy sets theory, Lee (2000) introduced the positive membership function $\mu_{A}^{+}(x)$ and the negative membership function $v_{A}^{-}(x)$, with the following conditions: $\mu_{A}^{+}(x) \in[0,1]$ and $v_{A}^{-}(x) \in[-1,0]$.

In intuitionistic fuzzy sets, Atanassov (1986) introduced the membership $T_{A}(x)$ and non-membership $F_{A}(x)$ functions, with the following conditions: $T_{A}(x), F_{A}(x) \in[0,1]$ and $T_{A}(x)+F_{A}(x) \leq 1$. In intuitionistic fuzzy sets, indeterminacy $\pi_{A}(x)$ is $1-T_{A}(x)-F_{A}(x)$ by default.

The condition $T_{A}(x)+F_{A}(x) \leq 1$ can be a limitation for using intuitionistic fuzzy numbers. Therefore, in Pythagorean fuzzy sets, this condition is transformed into the following $T_{A}(x)^{2}+F_{A}(x)^{2} \leq 1$ in order to facilitate their use in relation to intuitionistic fuzzy sets.

Smarandache (1999) proposed neutrosophic sets, as the generalization of fuzzy sets and their extensions. Neutrosophic sets have three independent membership functions, namely: the truth-membership function $T_{A}(x)$, the falsitymembership $F_{A}(x)$ function and the indeterminacy-membership $I_{A}(x)$ function. Smarandache (1999) and Wang et al. (2010) further proposed a single-valued neutrosophic set by modifying the conditions $T_{A}(x), I_{A}(x)$ and $F_{A}(x) \in[0,1]$ and 
Multiple-criteria Decision-making Based on the Use of Single-valued Neutrosophic Sets and Similarity Measures

$0 \leq T_{A}(x)+I_{A}(x)+F_{A}(x) \leq 3$, which are more suitable for solving scientific and engineering problems ( $\mathrm{Li}$ et al., 2016).

Single-valued neutrosophic numbers are used for solving a number of different problems, as a result of which many approaches have been proposed for their ranking, such as ranking based on the score and accuracy functions (Sahin 2014), ranking based on the Euclidean and Hamming distances (Ye, 2015; Pramanik et al., 2015), ranking based on different similarity measures (Ye, 2014).

There are a number of the similarity measures that can be used to rank singlevalued neutrosophic numbers. Therefore, an overview of some prominent similarity measures is given below, and the rest of the paper is organized in the following way: in Section 2, some basic concepts of neutrosophic sets and singlevalued neutrosophic numbers are presented; in Section 3, some distances and similarity measures between $\mathrm{n}$-dimensional vectors are considered, whereas in Section 3.1, similarity measures between two single-valued neutrosophic numbers are given; in Section 4, approaches for ranking of single-valued neutrosophic numbers are presented; in Section 5, the evaluation of alternatives by using single valued neutrosophic numberss is given; in Section 6, a group multi-criteria decision-making approach based on the use of single-valued neutrosophic numbers and similarity measures is presented, and in Section 7, an example is considered with the aim of explaining the proposed methodology in detail. Section 8 is a presentation of the analysis conducted and the conclusions of the paper are given in the last section.

\section{Some Basic Concepts of Neutrosophic Sets and Single-Valued Neutrosophic Numbers}

Definition 1. Let $X$ be the universe of discourse, with a generic element in $X$ denoted by $x$ (Smarandache, 1999). The Neutrosophic Set (NS) $A$ in $X$ is an object having the following form:

$$
A=\left\{\left\langle x, T_{A}(x), I_{A}(x), F_{A}(x)\right\rangle \mid x \in X\right\},
$$

with: $\left.\quad T_{A}: X \rightarrow\right]^{-} 0,1^{+}\left[; \quad I_{A}: X \rightarrow\right]^{-} 0,1^{+}\left[; \quad F_{A}: X \rightarrow\right]^{-} 0,1^{+}[\quad$ and ${ }^{-} 0 \leq T_{A}(x)+I_{A}(x)+F_{A}(x) \leq 3^{+}$, where: $T_{A}(x), \quad I_{A}(x)$ and $F_{A}(x)$ are the truthmembership function, the indeterminacy-membership function, and the falsitymembership function, respectively.

Definition 2. Let $X$ be a nonempty set. The Single-Valued Neutrosophic Set (SVNS) $A$ in $X$ is as follows (Wang et al., 2010):

$$
A=\left\{\left\langle x, T_{A}(x), I_{A}(x), F_{A}(x)\right\rangle \mid x \in X\right\},
$$

DOI: 10.24818/18423264/55.2.21.01 
Dragisa Stanujkic, Darjan Karabasevic, Gabrijela Popovic, Florentin Smarandache, Edmundas Kazimieras Zavadskas, Ieva Meidutè-Kavaliauskienè

with: $T_{A}: X \rightarrow[0,1] ; I_{A}: X \rightarrow[0,1] ; F_{A}: X \rightarrow[0,1]$ and

$0 \leq T_{A}(x)+I_{A}(x)+F_{A}(x) \leq 3$, where: $T_{A}(x), I_{A}(x)$ and $F_{A}(x)$ are the truthmembership function, the intermediacy-membership function, and the falsitymembership function, respectively, $T_{A}, I_{A}, F_{A}: X \rightarrow[0,1]$ and $0 \leq T_{A}(x)+I_{A}(x)+F_{A}(x) \leq 3$.

The truth-membership, indeterminacy-membership, and falsitymembership functions are sometimes referred to as the truth, indeterminacy and falsity degrees, respectively.

Definition 3. A Single-Valued Neutrosophic Number (SVNN) $a=<t_{a}, i_{a}, f_{a}>$ is a special case of an SVNS on the set of real numbers $\mathfrak{R}$ where $t_{a}, i_{a}, f_{a} \in[0,1]$ and $0 \leq t_{a}+i_{a}+f_{a} \leq 3$ (Wang et al., 2010).

Definition 4. Let $x_{1}=<t_{1}, i_{1}, f_{1}>$ and $x_{2}=<t_{2}, i_{2}, f_{2}>$ be two SVNNs and $\lambda>0$. The basic operations over the two SVNNs are as follows (Smarandache, 1999):

$$
\begin{aligned}
& x_{1}+x_{2}=<t_{1}+t_{2}-t_{1} t_{2}, i_{1} i_{2}, f_{1} f_{2}>. \\
& x_{1} \cdot x_{2}=<t_{1} t_{2}, i_{1}+i_{2}-i_{1} i_{2}, f_{1}+f_{2}-f_{1} f_{2}>. \\
& \lambda x_{1}=<1-\left(1-t_{1}\right)^{\lambda}, i_{1}^{\lambda}, f_{1}^{\lambda}>. \\
& x_{1}^{\lambda}=<t_{1}^{\lambda}, i_{1}^{\lambda}, 1-\left(1-f_{1}\right)^{\lambda}>.
\end{aligned}
$$

Definition 5. Let $x=<t_{i}, i_{\mathrm{i}}, f_{i}>$ be an SVNN. The score function $s_{x}$ of $x$ is as follows (Sahin, 2014):

$$
s_{i}=\left(1+t_{i}-2 i_{i}-f_{i}\right) / 2,
$$

where $s_{i} \in[-1,1]$.

Definition 6. Let $a_{j}=<t_{j}, i_{\mathrm{j}}, f_{j}>$ be a collection of SVNSs and $W=\left(w_{1}, w_{2}, \ldots, w_{n}\right)^{T}$ be an associated weighting vector. The Single-Valued Neutrosophic Weighted Average (SVNWA) operator of $a_{j}$ is as follows (Sahin, 2014):

$$
\operatorname{SVNWA}\left(a_{1}, a_{2}, \ldots, a_{n}\right)=\sum_{j=1}^{n} w_{j} a_{j}=\left(1-\prod_{j=1}^{n}\left(1-t_{j}\right)^{w_{j}}, \prod_{j=1}^{n}\left(i_{j}\right)^{w_{j}}, \prod_{j=1}^{n}\left(f_{j}\right)^{w_{j}}\right),
$$

where: $w_{j}$ is the element $j$ of the weighting vector, $w_{j} \in[0,1]$ and $\sum_{j=1}^{n} w_{j}=1$.

Definition 7. Let $a_{j}=<t_{j}, i_{\mathrm{j}}, f_{j}>$ be a collection of SVNSs and $W=\left(w_{1}, w_{2}, \ldots, w_{n}\right)^{T}$ be an associated weighting vector. Then, the Single-Valued Neutrosophic Weighted Geometric (SVNWG) operator of $a_{j}$ is as follows (Sahin, 2014): 
Multiple-criteria Decision-making Based on the Use of Single-valued Neutrosophic Sets and Similarity Measures

$$
\operatorname{SVNWG}\left(a_{1}, a_{2}, \ldots, a_{n}\right)=\prod_{j=1}^{n}\left(a_{j}\right)^{w_{j}}=\left(\prod_{j=1}^{n}\left(t_{j}\right)^{w_{j}}, 1-\prod_{j=1}^{n}\left(1-i_{j}\right)^{w_{j}}, 1-\prod_{j=1}^{n}\left(1-f_{j}\right)^{w_{j}}\right),
$$

where: $w_{j}$ is the element $j$ of the weighting vector, $w_{j} \in[0,1]$ and $\sum_{j=1}^{n} w_{j}=1$

\section{Some Distances and Similarity Measures Between n-Dimensional Vectors}

In this section, the definitions of the two distances and the four similarity measures between two $n$-dimensional vectors are presented.

Definition 8. Let $X=\left(x_{1}, x_{2}, \ldots, x_{\mathrm{n}}\right)$ and $Y=\left(y_{1}, y_{2}, \ldots, y_{\mathrm{n}}\right)$ be two $n$ dimensional vectors, and $x_{i}, y_{i} \geq 0$.

The Hamming distance between $X$ and $Y$ is as follows:

$$
h_{(X, Y)}=\sum_{i=1}^{n}\left|x_{i}-y_{i}\right| \text {. }
$$

Definition 9. Let $X=\left(x_{1}, x_{2}, \ldots, x_{\mathrm{n}}\right)$ and $Y=\left(y_{1}, y_{2}, \ldots, y_{\mathrm{n}}\right)$ be two $n$ dimensional vectors, and $x_{i}, y_{i} \geq 0$. The Euclidean distance between $X$ and $Y$ is as follows:

$$
e_{(X, Y)}=\sqrt{\sum_{i=1}^{n}\left(x_{i}-y_{i}\right)^{2}} .
$$

Definition 10. Let $X=\left(x_{1}, x_{2}, \ldots, x_{\mathrm{n}}\right)$ and $Y=\left(y_{1}, y_{2}, \ldots, y_{\mathrm{n}}\right)$ be two $n$ dimensional vectors, and $x_{i}, y_{i} \geq 0$ (Dice, 1945). The Dice similarity measure of the two vectors $X$ and $Y$ is as follows:

$$
d_{(X, Y)}=\frac{2 X \cdot Y}{\|X\|_{2}^{2}+\|Y\|_{2}^{2}}=\frac{2 \sum_{i=1}^{n} x_{i} y_{i}}{\sum_{i=1}^{n} x_{i}^{2}+\sum_{i=1}^{n} y_{i}^{2}} .
$$

Definition 11. Let $X=\left(x_{1}, x_{2}, \ldots, x_{\mathrm{n}}\right)$ and $Y=\left(y_{1}, y_{2}, \ldots, y_{\mathrm{n}}\right)$ be two $n-$ dimensional vectors, and $x_{i}, y_{i} \geq 0$. The Jaccard similarity measure of the two vectors $X$ and $Y$ is as follows:

$$
j_{(X, Y)}=\frac{X \cdot Y}{\|X\|_{2}^{2}+\|Y\|_{2}^{2}-X \cdot Y}=\frac{\sum_{i=1}^{n} x_{i} y_{i}}{\sum_{i=1}^{n} x_{i}^{2}+\sum_{i=1}^{n} y_{i}^{2}+\sum_{i=1}^{n} x_{i} y_{i}} .
$$

Definition 12. Let $X=\left(x_{1}, x_{2}, \ldots, x_{\mathrm{n}}\right)$ and $Y=\left(y_{1}, y_{2}, \ldots, y_{\mathrm{n}}\right)$ be two $n$ dimensional vectors, and $x_{i}, y_{i} \geq 0$ (Salton \& McGill, 1983). The cosine similarity measure of the two vectors $X$ and $Y$ is as follows:

DOI: 10.24818/18423264/55.2.21.01 
Dragisa Stanujkic, Darjan Karabasevic, Gabrijela Popovic, Florentin Smarandache, Edmundas Kazimieras Zavadskas, Ieva Meidutè-Kavaliauskienė

$$
c_{(X, Y)}=\frac{X \cdot Y}{\|X\|_{2}\|Y\|_{2}}=\frac{\sum_{i=1}^{n} x_{i} y_{i}}{\sqrt{\sum_{i=1}^{n} x_{i}^{2}} \sqrt{\sum_{i=1}^{n} y_{i}^{2}}} .
$$

Definition 13. Let $X=\left(x_{1}, x_{2}, \ldots, x_{\mathrm{n}}\right)$ and $Y=\left(y_{1}, y_{2}, \ldots, y_{\mathrm{n}}\right)$ be two $n$ dimensional vectors, and $x_{i}, y_{i} \geq 0$ (Ye \& Zhang, 2014). The similarity measure based on the minimum and maximum operators of the two vectors $X$ and $Y$ is as follows:

$$
m_{(X, Y)}=\sum_{i=1}^{n} \frac{\min \left(x_{i}, y_{i}\right)}{\max \left(x_{i}, y_{i}\right)} .
$$

\subsection{The Similarity Measures of Two SVNNs}

In this subsection, the similarity measures of two SVNNs are presented. In addition, the similarity measures between an SVNN and an ideal point, defined as $\langle 1,0,0\rangle$, are also considered.

Definition 14. Let $x_{1}=<t_{1}, i_{1}, f_{1}>$ and $x_{2}=<t_{2}, i_{2}, f_{2}>$ be two SVNNs. The similarity measure based on the Hamming distance of the two SVNNs is as follows:

$$
h_{\left(x_{1}, x_{2}\right)}=1-\frac{1}{3}\left(\left|t_{1}-t_{2}\right|+\left|i_{1}-i_{2}\right|+\left|f_{1}-f_{2}\right|\right) .
$$

Definition 15. Let $x_{1}=<t_{1}, i_{1}, f_{1}>$ and $x_{2}=<t_{2}, i_{2}, f_{2}>$ be two SVNNs. The similarity measure based on the Euclidean distance of the two SVNNs is as follows:

$$
e_{\left(x_{1}, x_{2}\right)}=1-\left(\frac{1}{3}\left(\left|t_{1}-t_{2}\right|^{2}+\left|i_{1}-i_{2}\right|^{2}+\left|f_{1}-f_{2}\right|^{2}\right)\right)^{\frac{1}{2}} .
$$

Definition 16. Let $x_{1}=<t_{1}, i_{1}, f_{1}>$ and $x_{2}=<t_{2}, i_{2}, f_{2}>$ be two SVNNs (Ye, 2014). The Dice similarity measure of the two SVNNs is as follows:

$$
d_{\left(x_{1}, x_{2}\right)}=\frac{2\left(t_{1} t_{2}+i_{1} i_{2}+f_{1} f_{2}\right)}{\left(t_{1}^{2}+i_{1}^{2}+f_{1}^{2}\right)+\left(t_{2}^{2}+i_{2}^{2}+f_{2}^{2}\right)} .
$$

Definition 17. Let $x_{1}=<t_{1}, i_{1}, f_{1}>$ and $x_{2}=<t_{2}, i_{2}, f_{2}>$ be two SVNNs (Ye, 2014). The Jaccard similarity measure of the two SVNNs is as follows:

$$
j_{\left(x_{1}, x_{2}\right)}=\frac{t_{1} t_{2}+i_{1} i_{2}+f_{1} f_{2}}{\left(t_{1}^{2}+i_{1}^{2}+f_{1}^{2}\right)+\left(t_{2}^{2}+i_{2}^{2}+f_{2}^{2}\right)+\left(t_{1} t_{2}+i_{1} i_{2}+f_{1} f_{2}\right)} .
$$

Definition 18. Let $x_{1}=<t_{1}, i_{1}, f_{1}>$ and $x_{2}=<t_{2}, i_{2}, f_{2}>$ be two SVNNs (Ye, 2014). The cosine similarity measure of the two SVNNs is as follows: 
Multiple-criteria Decision-making Based on the Use of Single-valued Neutrosophic Sets and Similarity Measures

$$
c_{\left(x_{1}, x_{2}\right)}=\frac{t_{1} t_{2}+i_{1} i_{2}+f_{1} f_{2}}{\sqrt{t_{1}^{2}+i_{1}^{2}+f_{1}^{2}} \cdot \sqrt{t_{2}^{2}+i_{2}^{2}+f_{2}^{2}}} .
$$

Definition 19. Let $x_{1}=<t_{1}, i_{1}, f_{1}>$ and $x_{2}=<t_{2}, i_{2}, f_{2}>$ be two SVNNs (Ye $\&$ Zhang, 2014). The similarity measure based on the minimum and maximum operators of the two SVNNs is as follows:

$$
m_{\left(x_{1}, x_{2}\right)}=\frac{1}{3}\left(\frac{\min \left(t_{1}, t_{2}\right)}{\max \left(t_{1}, t_{2}\right)}+\frac{\min \left(i_{1}, i_{2}\right)}{\max \left(i_{1}, i_{2}\right)}+\frac{\min \left(f_{1}, f_{2}\right)}{\max \left(f_{1}, f_{2}\right)}\right) .
$$

\section{The Ranking of Single-Valued Neutrosophic Numbers}

There are several approaches to the comparison and ranking of SVNNs, such as: the ranking based on the use of the score function; the ranking based on distances from the ideal and anti-ideal points; and the ranking based on the use of similarity measures.

\subsection{The ranking based on the use of the score function}

The approach based on the use of the score function is commonly used. In this approach, the overall single-valued neutrosophic utility (OSVNU) of each alternative is first calculated by using Eq. (8), or Eq. (9), and then the score of each overall utility is determined by using Eq. (7). The alternative with a higher score is the most acceptable.Sahin (2014) and Mondal \& Pramanik (2014) can be mentioned as some examples of the ranking of alternatives based on the use of the score function.

\subsection{The ranking based on distances from the ideal and anti-ideal points}

The ranking of alternatives based on their distances to the ideal point and the anti-ideal point is mainly used in the neutrosophic extensions of the TOPSIS method. The ranking of alternatives in such approaches is based on the application of the following equation:

$$
c_{i}=\frac{d_{i}^{-}}{d_{i}^{+}+d_{i}^{-}},
$$

where: $c_{i}$ denotes the relative closeness to the ideal point of the alternative $i, d_{i}^{-}$and $d_{i}^{+}$denote the distance of the alternative $i$ to the anti-ideal point and the ideal point, respectively.

The alternative with the highest value of relative closeness is the most acceptable.

DOI: 10.24818/18423264/55.2.21.01 
Dragisa Stanujkic, Darjan Karabasevic, Gabrijela Popovic, Florentin Smarandache, Edmundas Kazimieras Zavadskas, Ieva Meidutè-Kavaliauskienė

Ye (2015), Pramanik et al. (2015) and Pouresmaeil et al. (2017) used the neutrosophic extensions of the TOPSIS method to solve different decision-making problems, such as the selection of the best investment alternative, the recruitment of an assistant teacher, and so forth. In these extensions of the use of both, the Euclidean and the Hamming distances are considered.

Biswas et al. (2016) used the neutrosophic extension of the TOPSIS method to select the most suitable tablet. In this approach, the Euclidean distance between two SVNNs was used.

\subsection{The ranking based on the use of similarity measures}

This approach is based on the similarity between the single-valued neutrosophic overall utility of an alternative and the single-valued neutrosophic ideal point. In a fashion similar to the case of the ranking based on the score function, the overall single-valued neutrosophic utility of each alternative is, first, calculated by using Eq. (8) or Eq. (9). Then, in the next step, the similarity measure between overall single-valued neutrosophic utility and the ideal point should be calculated. The alternative with the highest value of the similarity measure is the most acceptable.

The single-valued neutrosophic ideal point $r^{*}=<t^{*}, i^{*}, f^{*}>$ can be calculated as follows:

$$
r^{*}= \begin{cases}\left\langle\max _{i} t_{i}, \min _{i} i_{i}, \min _{i} f_{i}\right\rangle, & j \in \Omega_{\max }, \\ \left.\min _{i} t_{i}, \min _{i} i_{i}, \max _{i} f_{i}\right\rangle, & j \in \Omega_{\min }\end{cases}
$$

where $\Omega_{\max }$ and $\Omega_{\min }$ denote a set of beneficial and nonbeneficial criteria, respectively.

In cases when all criteria are beneficial, Eq. (24) is applied as follows:

$$
r^{*}=\left\langle\max _{i} t_{i j}, \min _{i} i_{i j}, \min _{i} f_{i j}\right\rangle,
$$

In neutrosophy, higher values for the truth degree and lower values for the falsity and indeterminacy degrees are preferable. Therefore, for the sake of simplicity, $r_{j}^{*}$ could be determined as follows:

$$
r^{*}=\langle 1,0,0\rangle \text {. }
$$

In the case of using Eq. (25) instead of Eq. (24), the previously defined Eqs. (16)-(21) have the following forms: 
Multiple-criteria Decision-making Based on the Use of Single-valued Neutrosophic Sets and Similarity Measures

Definition 20. Let $x=<t, i, f>$ be an SVNN and let the ideal point be defined as $\langle 1,0,0\rangle$. The similarity measure based on the Hamming distance of the SVNN $x$ and the ideal point is as follows:

$$
h_{(x)}=1-\frac{1}{3}\left(1-t_{x}+i_{x}+f_{x}\right) \text {. }
$$

Definition 21. Let $x=<t, i, f>$ be an SVNN and let the ideal point be defined as $\langle 1,0,0\rangle$. The similarity measure based on the Euclidean distance between the SVNN $x$ and the ideal point is as follows:

$$
e_{(x)}=1-\left(\frac{1}{3}\left[\left(1-t_{x}\right)^{2}+i_{x}^{2}+f_{x}^{2}\right]\right)^{\frac{1}{2}} .
$$

Definition 22. Let $x=<t, i, f>$ be an SVNN and let the ideal point be defined as $\langle 1,0,0\rangle$. The Dice similarity measure between the SVNN $x$ and the ideal point is as follows:

$$
d_{(x)}=\frac{2 t_{x}}{1+t_{x}^{2}+i_{x}^{2}+f_{x}^{2}} .
$$

Definition 23. Let $x=<t, i, f>$ be an SVNN and let the ideal point be defined as $\langle 1,0,0\rangle$. The Jaccard similarity measure between the SVNN $x$ and the ideal point is as follows:

$$
j_{(x)}=\frac{t_{x}}{1+t_{x}^{2}+i_{x}^{2}+f_{x}^{2}+t_{x}} .
$$

Definition 24. Let $x=<t, i, f>$ be an SVNN and let the ideal point be defined as $\langle 1,0,0\rangle$. The cosine similarity measure between the SVNN $x$ and the ideal point is as follows:

$$
c_{(x)}=\frac{t_{x}}{\sqrt{t_{x}^{2}+i_{x}^{2}+f_{x}^{2}}} .
$$

Definition 25. Let $x=<t, i, f>$ be an SVNN and let the ideal point be defined as $\langle 1,0,0\rangle$. The similarity measure based on the minimum and maximum operators between the SVNN $x$ and the ideal point is as follows:

$$
m_{(x)}=\frac{1}{3} t_{x} .
$$

The following can be mentioned as some examples of the ranking of alternatives based on the use of similarity measures: Ye (2014) used three similarity measures, i.e. the Jaccard, Dice, and cosine similarity measures, to select

\section{DOI: 10.24818/18423264/55.2.21.01}


Dragisa Stanujkic, Darjan Karabasevic, Gabrijela Popovic, Florentin Smarandache, Edmundas Kazimieras Zavadskas, Ieva Meidutè-Kavaliauskienè

the best investment alternative. Ye (2017) also used the cosine similarity measure for the fault diagnosis of a steam turbine. Chen et al. (2017) used similarity measures between two SVNNs to select the most appropriate construction project.

\section{The Evaluation of Alternatives by Using SVNNs}

The evaluation of alternatives in relation to evaluation criteria is a very significant step, especially so when interviewing the respondents who are unfamiliar with using neutrosophic numbers.

One possible way to perform evaluation more efficiently and more accurately is to form one statement sentence for each criterion, thus enabling the respondent to express his or her opinion regarding its accuracy and/or inaccuracy. For example, the following sentences can be used for the criteria delivery and price: The supplier's delivery time is timely and the supplier provides fair prices.

The values obtained in such a manner represent the truth degree and/or the falsity degree. In addition, the respondent may also express his or her uncertainty about the given answers. In such a case, the information obtained in that manner belongs to the indeterminacy degree. In some cases, the respondent may only use the truth degree, and then the falsity degree and the indeterminacy degree are set to zero.

Two linguistic scales were suggested in order to facilitate the evaluation of the alternatives. The first linguistic scale, shown in Table 1, can be used to express the level of the accuracy and inaccuracy of a statement sequence, while the second, shown in Table 2, can be used to express uncertainty about the given answers.

Table 1. The linguistic scale for expressing the level of accuracy and inaccuracy

\begin{tabular}{lccc}
\hline Linguistic variable & $\mathbf{0} \div \mathbf{1}$ & $\mathbf{0} \div \mathbf{1 0}$ & $\mathbf{0} \div \mathbf{1 0 0 \%}$ \\
\hline Extremely satisfied & $\approx 1.00$ & $\approx 10$ & $\approx 100 \%$ \\
Very satisfied & $\approx 0.75$ & $\approx 7.5$ & $\approx 75 \%$ \\
Moderately & $\approx 0.50$ & $\approx 5.0$ & $\approx 50 \%$ \\
satisfied & $\approx 0.25$ & $\approx 2.5$ & $\approx 25 \%$ \\
Slightly satisfied & $\approx 0.00$ & $\approx 0.0$ & $\approx 0 \%$ \\
Dissatisfied & &
\end{tabular}

Table 2. The linguistic scale for expressing the level of uncertainty about the given answers 
Multiple-criteria Decision-making Based on the Use of Single-valued Neutrosophic Sets and Similarity Measures

\begin{tabular}{lccc}
\hline Linguistic variable & $\mathbf{0} \div \mathbf{1}$ & $\mathbf{0} \div \mathbf{1 0}$ & $\mathbf{0} \div \mathbf{1 0 0 \%}$ \\
\hline Unconvinced & $\approx 1.00$ & $\approx 10$ & $\approx 100 \%$ \\
Poorly convinced & $\approx 0.75$ & $\approx 7.5$ & $\approx 75 \%$ \\
Moderately & $\approx 0.50$ & $\approx 5.0$ & $\approx 50 \%$ \\
convinced & $\approx 0.25$ & $\approx 2.5$ & $\approx 25 \%$ \\
Convinced & $\approx 0.0$ & $\approx 0 \%$ \\
\hline
\end{tabular}

The linguistic variables from both tables can be transformed into any of the proposed ranges, depending on the respondents' preferences, but the first interval 0 $\div 1$ does not require normalization, whereas in the case of the second and the third ranges, the values should be transformed into the interval $[0,1]$. The use of linguistic variables may make it easier for respondents to perform an evaluation, but in some ways, they can restrict the precise expression of their attitudes, which is why respondents should be informed that the given values are only approximate and that they may use any value from the interval $[0,1]$, or other scopes.

\section{A Group Multi-Criteria Decision-Making Approach Based on Single-Valued Neutrosophic Numbers and Similarity Measures}

The procedure for solving a multi-criteria decision-making problem that contains the $m$ alternatives that are evaluated based on $n$ criteria by $K$ experts using SVNNs can precisely be expressed by the following algorithm: alternatives.

Step 1. Define the goal of the evaluation and identify available

Step 2. Form a group of experts who will perform the evaluation.

Step 3. Define a set of evaluation criteria and determine their significance, i.e. criteria weights. in Section 5.

Step 4. Form a questionnaire and define an evaluation scale, as discussed

Step 5. Evaluate the alternatives in relation to the selected criteria. (9).

Step 6. Construct a group decision-making matrix by using Eq. (8) or Eq.

Step 7. Calculate the single-valued neutrosophic overall utility of the alternatives by using Eq. (8) or Eq. (9).

Step 8. Determine the single-valued neutrosophic ideal point by using Eq. (24) or Eq. (25).

Step 9. Calculate a similarity measure for each alternative. Similarity measures can be calculated by applying any of Eqs (16)-(21) if the ideal point is determined by using Eq. (24), or by applying any of Eqs (26)-(31) if the ideal point is determined by using Eq. (25).

Step 10. Rank the alternatives and/or select the best one. The alternative with a higher similarity measure is more preferable.

DOI: 10.24818/18423264/55.2.21.01 
Dragisa Stanujkic, Darjan Karabasevic, Gabrijela Popovic, Florentin Smarandache, Edmundas Kazimieras Zavadskas, Ieva Meidutè-Kavaliauskienė

\section{A Numerical Illustration}

In order to briefly demonstrate the usability of SVNNs for solving MCDM problems, an example of the supplier selection is presented in this section.

Allow us to assume that one company must consider engaging a new supplier. Therefore, a team of three experts is formed with the aim of selecting the most appropriate supplier from the four alternatives denoted as $A_{1}-A_{4}$ based on the following criteria: C1 - Delivery; C2 - Quality; C3 - Flexibility; C4 - Service; and C5 - Price.

The set of the given criteria is determined based on the paper by Chang et al. (2011), who performed an evaluation of the criteria in order to determine which of the criteria are the most influential in the case of the supplier selection. As a result, the five criteria used in our case are singled out as the most significant.

The ratings obtained from the three experts, after their transformation into SVNNs and corrections, are presented in Tables 3, 4 and 5.

Table 3. The ratings obtained from the first of the three experts

\begin{tabular}{|c|c|c|c|c|c|}
\hline & $C_{1}$ & $C_{2}$ & $C_{3}$ & $C_{4}$ & $C_{5}$ \\
\hline$A_{1}$ & $\langle 0.8,0.30,0.10\rangle$ & $\langle 0.7,0.0,0.3\rangle$ & $\langle 0.6,0.0,0.4\rangle$ & $\langle 0.7,0.0,0.3\rangle$ & $\langle 0.5,0.0,0.5\rangle$ \\
\hline$A_{2}$ & $<0.7,0.00,0.20\rangle$ & $\langle 0.8,0.0,0.2\rangle$ & $\langle 0.8,0.0,0.2\rangle$ & $<0.8,0.0,0.2>$ & $\langle 0.8,0.0,0.2\rangle$ \\
\hline$A_{3}$ & $\langle 0.5,0.00,0.20\rangle$ & $\langle 0.5,0.0,0.5>$ & $\langle 0.6,0.0,0.4>$ & $\langle 0.6,0.0,0.4>$ & $\langle 0.7,0.0,0.3\rangle$ \\
\hline$A_{4}$ & $\langle 0.5,0.20,0.20\rangle$ & $\langle 0.5,0.2,0.5\rangle$ & $\langle 0.6,0.2,0.4\rangle$ & $\langle 0.6,0.2,0.4\rangle$ & $\langle 0.7,0.2,0.3\rangle$ \\
\hline
\end{tabular}

Table 4. The ratings obtained from the second of the three experts

\begin{tabular}{|c|c|c|c|c|}
\hline$C_{1}$ & $C_{2}$ & $C_{3}$ & $C_{4}$ & $C_{5}$ \\
\hline$A_{1}\langle 0.6,0.00,0.40\rangle$ & $\langle 0.7,0.0,0.3>$ & $\langle 0.6,0.0,0.4\rangle$ & $\langle 0.5,0.0,0.0\rangle$ & $\langle 0.6,0.0,0.0\rangle$ \\
\hline$A_{2}\langle 0.8,0.00,0.20\rangle$ & $<0.6,0.0,0.4>$ & $\langle 0.7,0.0,0.3\rangle$ & $\langle 0.8,0.0,0.2>$ & $\langle 0.6,0.0,0.0\rangle$ \\
\hline$A_{3}\langle 0.7,0.00,0.30\rangle$ & $\langle 0.8,0.0,0.2\rangle$ & $\langle 0.7,0.0,0.3\rangle$ & $\langle 0.6,0.0,0.4>$ & $\langle 0.7,0.0,0.3>$ \\
\hline$\langle 0.7,0.20,0.30\rangle$ & $\langle 0.8,0.2,0.2\rangle$ & $\langle 0.7,0.2,0.3\rangle$ & $\langle 0.6,0.2,0.4\rangle$ & $\langle 0.7,0.2,0.3\rangle$ \\
\hline
\end{tabular}

Table 5. The ratings obtained from the third of the three experts

\begin{tabular}{cccccc}
\hline $\boldsymbol{C}_{\mathbf{1}}$ & $\boldsymbol{C}_{\mathbf{2}}$ & $\boldsymbol{C}_{\mathbf{3}}$ & $\boldsymbol{C}_{\mathbf{4}}$ & $\boldsymbol{C}_{\mathbf{5}}$ \\
\hline$A_{1}$ & $\langle 0.8,0.50,0.20\rangle$ & $\langle 0.6,0.5,0.4\rangle$ & $\langle 0.5,0.5,0.5\rangle$ & $\langle 0.6,0.5,0.4\rangle$ & $\langle 0.8,0.5,0.2\rangle$ \\
$A_{2}$ & $\langle 0.6,0.20,0.10\rangle$ & $\langle 0.6,0.2,0.4\rangle$ & $\langle 0.8,0.2,0.2\rangle$ & $\langle 0.5,0.2,0.5\rangle$ & $\langle 0.7,0.2,0.3\rangle$ \\
$A_{3}$ & $\langle 0.6,0.10,0.40\rangle$ & $\langle 0.7,0.1,0.3\rangle$ & $\langle 0.6,0.1,0.2\rangle$ & $\langle 0.6,0.1,0.4\rangle$ & $\langle 0.5,0.1,0.5\rangle$ \\
$A_{4}$ & $\langle 0.6,0.20,0.40\rangle$ & $\langle 0.7,0.2,0.3\rangle$ & $\langle 0.6,0.2,0.2\rangle$ & $\langle 0.6,0.2,0.4\rangle$ & $\langle 0.5,0.2,0.5\rangle$ \\
\hline
\end{tabular}

As shown in Step 5 of the previously presented procedure for solving multi-criteria decision-making problems, the group decision-making matrix shown in Table 6 was constructed by using Eq. (8). In this calculation, all the three experts had the same significance, i.e. $w_{j}=0.333 ; j=1,2$ and 3 . 
Multiple-criteria Decision-making Based on the Use of Single-valued Neutrosophic Sets and Similarity Measures

\begin{tabular}{cccccc}
\hline \multicolumn{6}{c}{ Table 6. The group decision-making matrix } \\
\hline $\boldsymbol{C}_{\mathbf{1}}$ & $\boldsymbol{C}_{\mathbf{2}}$ & $\boldsymbol{C}_{\mathbf{3}}$ & $\boldsymbol{C}_{\mathbf{4}}$ & $\boldsymbol{C}_{\mathbf{5}}$ \\
\hline$A_{1}$ & $\langle 0.75,0.0,0.24\rangle$ & $\langle 0.67,0.0,0.33\rangle$ & $\langle 0.57,0.0,0.44\rangle$ & $\langle 0.61,0.0,0.25\rangle$ & $\langle 0.66,0.0,0.26\rangle$ \\
$A_{2}$ & $\langle 0.71,0.0,0.17\rangle$ & $\langle 0.68,0.0,0.34\rangle$ & $\langle 0.77,0.0,0.23\rangle$ & $\langle 0.73,0.0,0.32\rangle$ & $\langle 0.71,0.0,0.18\rangle$ \\
$A_{3}$ & $\langle 0.61,0.0,0.30\rangle$ & $\langle 0.69,0.0,0.35\rangle$ & $\langle 0.64,0.0,0.30\rangle$ & $\langle 0.60,0.0,0.40\rangle$ & $\langle 0.64,0.0,0.37\rangle$ \\
$A_{4}$ & $\langle 0.75,0.0,0.24\rangle$ & $\langle 0.67,0.0,0.33\rangle$ & $\langle 0.57,0.0,0.44\rangle$ & $\langle 0.61,0.0,0.25\rangle$ & $\langle 0.66,0.0,0.26\rangle$ \\
\hline
\end{tabular}

In the next step, Step 6 of the above-proposed procedure, the overall ratings were calculated by using Eq. (7) and the following weights $w_{j}=\{0.18,0.21$, $0.20,0.18,0.23\}$. The overall ratings are shown in Table 7 .

Table 7. The overall single-valued neutrosophic utility of the alternatives

\begin{tabular}{lc}
\hline & Overall utility \\
\hline$A_{1}<0.65,0.00,0.30>$ \\
$A_{2}<0.72,0.00,0.24>$ \\
$A_{3}<0.64,0.00,0.34>$ \\
$A_{4}<0.65,0.00,0.30>$ \\
\hline
\end{tabular}

Finally, the ranking results obtained by using all the approaches considered in Section 3 and the ideal point $r^{*}=<0.72,0.00,0.24>$, calculated by using Eq. (24), are demonstrated in Table 8.

Table 8. The ranking results obtained by using the considered approaches

\begin{tabular}{|c|c|c|c|c|c|c|c|c|c|c|c|c|c|c|}
\hline & \multicolumn{2}{|c|}{ I } & \multicolumn{2}{|c|}{ II } & \multicolumn{2}{|c|}{ III } & \multicolumn{2}{|l|}{ IV } & \multicolumn{2}{|l|}{$\mathbf{V}$} & \multicolumn{2}{|l|}{ VI } & \multicolumn{2}{|c|}{ VII } \\
\hline & $h_{(i)}$ & & $e_{(i)}$ & & $d_{(i)}$ & & $j_{(i)}$ & & $c_{(i)}$ & & $m m_{(i)}$ & & $S_{(i)}$ & \\
\hline$A_{1}$ & 0.89 & 3 & 0.79 & 3 & 0.96 & 3 & 0.325 & 3 & 0.963 & 3 & 0.56 & 3 & 0.46 & 3 \\
\hline$A_{2}$ & 0.99 & 1 & 0.96 & 1 & 1.00 & 1 & 0.333 & 1 & 0.999 & 1 & 0.67 & 1 & 0.67 & 1 \\
\hline$A_{3}$ & 0.94 & 2 & 0.86 & 2 & 0.98 & 2 & 0.330 & 2 & 0.984 & 2 & 0.52 & 4 & 0.61 & 2 \\
\hline$A_{4}$ & 0.88 & 4 & 0.79 & 4 & 0.96 & 4 & 0.324 & 4 & 0.960 & 4 & 0.58 & 2 & 0.45 & 4 \\
\hline
\end{tabular}

As can be seen from Table 8, all the considered approaches provide almost the same ranking orders of the evaluated alternatives. One discrepancy occurs when using the similarity measure based on the minimum and maximum operators of the two SVNNs.

In Column I of Table 8, the ranking results obtained by using the score function calculated by using Eq. (7) are shown. As can be seen, the ranking orders obtained by using the score function and the considered similarity measures are the same, except for the ranking order obtained by using the similarity measure based on the minimum and maximum operators of the two SVNNs. The obtained ranking orders of the alternatives are shown in Table 9.

Table 9. The ranking orders of the alternatives obtained by using the considered approaches

\begin{tabular}{lllllll}
\hline $\boldsymbol{h}_{(i)}$ & $\boldsymbol{e}_{(i)}$ & $\boldsymbol{d}_{(i)}$ & $\boldsymbol{j}_{(i)}$ & $\boldsymbol{c}_{(i)}$ & $\boldsymbol{m m}_{(i)}$ & $\boldsymbol{S}_{(i)}$ \\
\hline
\end{tabular}

DOI: 10.24818/18423264/55.2.21.01 
Dragisa Stanujkic, Darjan Karabasevic, Gabrijela Popovic, Florentin Smarandache, Edmundas Kazimieras Zavadskas, Ieva Meidutè-Kavaliauskienė

\begin{tabular}{lllllllll}
\hline$A_{1}$ & 3 & 3 & 3 & 3 & 3 & 3 & 3 \\
$A_{2}$ & 1 & 1 & 1 & 1 & 1 & 1 & 1 \\
$A_{3}$ & 2 & 2 & 2 & 2 & 2 & 4 & 2 \\
$A_{4}$ & 4 & 4 & 4 & 4 & 4 & 2 & 4 \\
\hline
\end{tabular}

The similar ranking results are obtained in the case when the ideal point $r^{*}=<1.00,0.00,0.00>$ was used. The ranking results obtained in that way are shown in Table 10.

Table 10. The ranking results obtained by using the considered approaches

\begin{tabular}{|c|c|c|c|c|c|c|c|c|c|c|c|c|c|c|}
\hline & \multicolumn{2}{|c|}{ I } & \multicolumn{2}{|c|}{ II } & \multicolumn{2}{|c|}{ III } & \multicolumn{2}{|l|}{ IV } & \multicolumn{2}{|c|}{$\mathbf{V}$} & \multicolumn{2}{|c|}{ VI } & \multicolumn{2}{|c|}{ VII } \\
\hline & $h_{(i)}$ & & $e_{(i)}$ & & $d_{(i)}$ & & $j_{(i)}$ & & $c_{(i)}$ & & $m m_{(i)}$ & & $s_{(i)}$ & \\
\hline$A_{1}$ & 0.71 & 3 & 0.49 & 3 & 0.84 & 3 & 0.29 & 3 & 0.87 & 3 & 0.22 & 2 & 0.46 & 3 \\
\hline$A_{2}$ & 0.80 & 1 & 0.63 & 1 & 0.91 & 1 & 0.31 & 1 & 0.95 & 1 & 0.24 & 1 & 0.67 & 1 \\
\hline$A_{3}$ & 0.75 & 2 & 0.50 & 2 & 0.84 & 2 & 0.29 & 2 & 0.88 & 2 & 0.21 & 3 & 0.61 & 2 \\
\hline$A_{4}$ & 0.70 & 4 & 0.46 & 4 & 0.82 & 4 & 0.29 & 4 & 0.85 & 4 & 0.21 & 3 & 0.45 & 4 \\
\hline
\end{tabular}

The obtained ranking orders are the same as those in the previous case, and the only discrepancy occurs when using the similarity measure based on the minimum and maximum operators of the two SVNNs.

\section{The Analysis}

To determine whether all the considered similarity measures generate the same or similar ranking orders of the alternatives, one theoretical case of the ranking in which the overall utility of the alternatives is represented by certain characteristic SVNNs is considered below, as is shown in Table 11.

Table 11. The overall utility of the alternatives

$$
\begin{array}{ll}
\hline \boldsymbol{A}_{1} & <1.00,0.00,0.00> \\
\boldsymbol{A}_{\mathbf{2}} & <1.00,0.00,1.00> \\
\boldsymbol{A}_{3} & <1.00,1.00,0.00> \\
\boldsymbol{A}_{\mathbf{4}} & <1.00,1.00,1.00> \\
\boldsymbol{A}_{5} & <0.00,0.00,0.00> \\
\boldsymbol{A}_{6} & <0.00,0.00,1.00> \\
\boldsymbol{A}_{7} & <0.00,1.00,0.00> \\
\boldsymbol{A}_{\mathbf{8}} & <0.00,1.00,1.00> \\
\hline \boldsymbol{r}^{*} & <1.00,0.00,0.00> \\
\hline
\end{array}
$$

The reference point determined by using Eq. (24) is also shown in Table 11. The calculation details are presented in Table 12. 
Multiple-criteria Decision-making Based on the Use of Single-valued Neutrosophic Sets and Similarity Measures

\begin{tabular}{cccccccccccccccc}
\hline \multicolumn{1}{c}{ Table 12. The ranking results obtained by using the considered approaches } \\
\hline \multicolumn{4}{c}{ I } & \multicolumn{1}{c}{ II } & \multicolumn{1}{c}{ III } & \multicolumn{1}{c}{ IV } & \multicolumn{3}{c}{ V } & \multicolumn{3}{c}{ VI } & \multicolumn{2}{c}{ VII } \\
\hline & $h_{(i)}$ & & $e_{(i)}$ & & $d_{(i)}$ & & $j_{(i)}$ & & $c_{(i)}$ & $m m_{(i)}$ & & $s_{(i)}$ & \\
\hline$A_{1}$ & 1.00 & 1 & 1.00 & 1 & 1.00 & 1 & 0.33 & 1 & 1.00 & 1 & 0.33 & 1 & 1.00 & 1 \\
$A_{2}$ & 0.67 & 2 & 0.42 & 2 & 0.67 & 2 & 0.25 & 2 & 0.71 & 2 & 0.33 & 1 & 0.50 & 2 \\
$A_{3}$ & 0.67 & 2 & 0.42 & 2 & 0.67 & 2 & 0.25 & 2 & 0.71 & 2 & 0.33 & 1 & 0.00 & 4 \\
$A_{4}$ & 0.33 & 5 & 0.18 & 5 & 0.50 & 4 & 0.20 & 4 & 0.58 & 4 & 0.33 & 1 & -0.50 & 6 \\
$A_{5}$ & 0.67 & 2 & 0.42 & 2 & 0.00 & 5 & 0.00 & 5 & 0.00 & 5 & 0.00 & 5 & 0.50 & 2 \\
$A_{6}$ & 0.33 & 5 & 0.18 & 5 & 0.00 & 5 & 0.00 & 5 & 0.00 & 5 & 0.00 & 5 & 0.00 & 4 \\
$A_{7}$ & 0.33 & 5 & 0.18 & 5 & 0.00 & 5 & 0.00 & 5 & 0.00 & 5 & 0.00 & 5 & -0.50 & 6 \\
$A_{8}$ & 0.00 & 8 & 0.00 & 8 & 0.00 & 5 & 0.00 & 5 & 0.00 & 5 & 0.00 & 5 & -1.00 & 8 \\
\hline
\end{tabular}

In order to make a comparison, the value for the alternative $A_{5}$ obtained by using the cosine similarity measure is set to zero, because this similarity measure cannot be used when all the three membership functions are equal to zero. The obtained ranking results are shown in Table 13 and in Figure 1.

Table 13. The ranking orders of the alternatives obtained by using the considered approaches

\begin{tabular}{|c|c|c|c|c|c|c|c|}
\hline & $\boldsymbol{h}_{(i)}$ & $\boldsymbol{e}_{(i)}$ & $d_{(i)}$ & $j_{(i)}$ & $\boldsymbol{c}_{(i)}$ & $\boldsymbol{m m}_{(i)}$ & $s_{(i)}$ \\
\hline$A_{1}$ & 1 & 1 & 1 & 1 & 1 & 1 & 1 \\
\hline$A_{2}$ & 2 & 2 & 2 & 2 & 2 & 1 & 2 \\
\hline$A_{3}$ & 2 & 2 & 2 & 2 & 2 & 1 & 4 \\
\hline$A_{4}$ & 5 & 5 & 4 & 4 & 4 & 1 & 6 \\
\hline$A_{5}$ & 2 & 2 & 5 & 5 & 5 & 5 & 2 \\
\hline$A_{6}$ & 5 & 5 & 5 & 5 & 5 & 5 & 4 \\
\hline$A_{7}$ & 5 & 5 & 5 & 5 & 5 & 5 & 6 \\
\hline$A_{8}$ & 8 & 8 & 5 & 5 & 5 & 5 & 8 \\
\hline $\max$ & 8 & 8 & 5 & 5 & 5 & 5 & 8 \\
\hline
\end{tabular}

Table 13 shows that the alternative $A_{1}$ is the best-ranked and the alternative $A_{8}$ is the worst-ranked in all the approaches. However, the ranking order of the other alternatives is slightly different. 
Dragisa Stanujkic, Darjan Karabasevic, Gabrijela Popovic, Florentin Smarandache, Edmundas Kazimieras Zavadskas, Ieva Meidutè-Kavaliauskienė

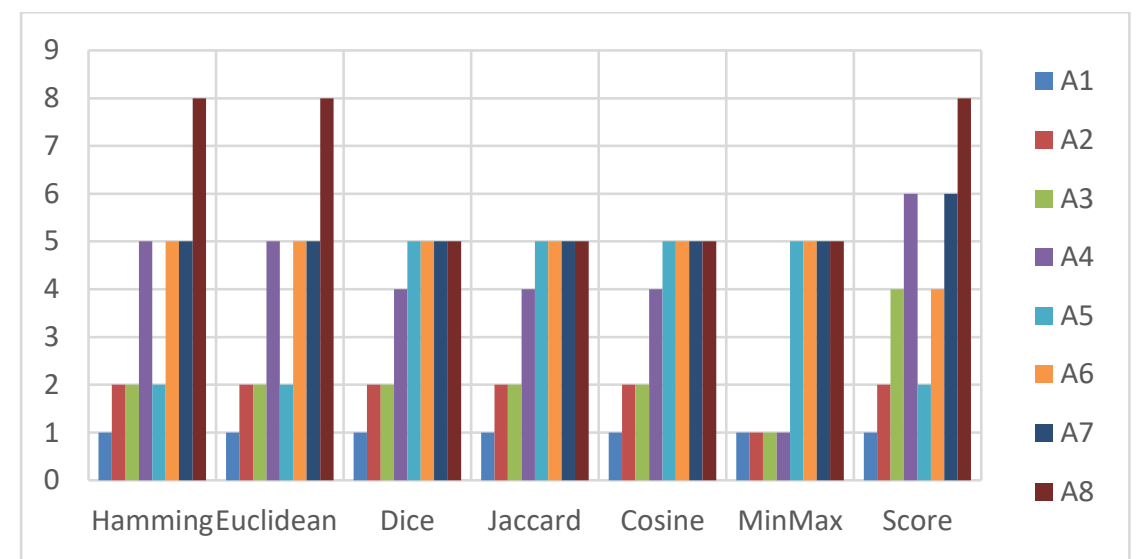

Figure 1. The ranking differences as a result of applying different approaches

The largest discrepancy can be observed in the ranking obtained by using the similarity measure based on the minimum and maximum operators, whereas the highest similarity can be observed in the results obtained by using the Dice, Jaccard and Cosine measures. A high similarity can also be observed in the results obtained by applying the similarity measures based on the Hamming and Euclidean distances.

However, it should be noted that, in this example, the overall significance of the considered alternatives varied from ideally good to ideally bad. In real cases, it can be expected that differences will be less noticeable.

\section{Conclusions}

Neutrosophic sets extend the concept proposed in fuzzy and intuitionistic fuzzy sets. These sets enable us to use the three independent membership functions: the truth function, the intermediacy function and the falsity membership function. Therefore, neutrosophic sets can be very useful for solving complex decision-making problems and/or problems related to predictions and uncertainty.

In multi-criteria decision-making, neutrosophic sets enable the evaluation based on the use of a smaller number of more complex evaluation criteria. Despite their complexity, by using appropriate questionnaires, they can be used for an efficient evaluation of alternatives in relation to a set of evaluation criteria.

As a result of applying neutrosophic sets in solving numerous decisionmaking problems, a few approaches to the ranking of neutrosophic numbers based on similarity measures, as well as a few other, are proposed. Several prominent and less prominent similarity measures, such as the Dice, Jaccard and cosine similarity measures, measure similarity based on the minimum and maximum operators, and the similarity measures derived from the Hamming and Euclidean distances are presented and their use is considered in the case of the two numerical illustrations. 
Multiple-criteria Decision-making Based on the Use of Single-valued Neutrosophic Sets and Similarity Measures

One additional analysis was also carried out so as to determine whether similarity measures always generate the same or similar ranking orders of alternatives.

The obtained results indicate a significant similarity between the results obtained by using certain similarity measures, simultaneously also indicating that in certain cases there are evident differences.

\section{REFERENCES}

[1] Atanassov, K.T. (1986), Intuitionistic Fuzzy Sets. Fuzzy Sets and Systems, 20(1): 87-96;

[2] Atanassov, K., Gargov, G. (1989), Interval Valued Intuitionistic Fuzzy Sets. Fuzzy Sets and Systems, 31(3): 343-349;

[3] Biswas, P., Pramanik, S., Giri, B.C. (2016), TOPSIS Method for MultiAttribute Group Decision-Making under Single-Valued Neutrosophic

Environment. Neural Computing and Applications, 27(3): 727-737;

[4] Chang, B., Chang, C.W., Wu, C. H. (2011), Fuzzy DEMATEL Method for

Developing Supplier Selection Criteria. Expert Systems with Applications, 38(3):

1850-1858;

[5] Chen, J., Ye, J., Du, S. (2017), Vector Similarity Measures between Refined Simplified Neutrosophic Sets and Their Multiple Attribute Decision-Making Method. Symmetry, 9(8): 153;

[6] Dice, L.R. (1945), Measures of the Amount of Ecologic Association between Species. Ecology, 26(3): 297-302;

[7] Lee K.M. (2000), Bipolar-valued Fuzzy Sets and their Basic Operations. In Proc. of International Conference on Intelligent Technologies, Bangkok, Thailand, 307-317;

[8] Li, Y., Liu, P., Chen, Y. (2016), Some Single Valued Neutrosophic Number Heronian Mean Operators and Their Application in Multiple Attribute Group Decision Making. Informatica, 27(1): 85-110;

[9] Mondal, K., Pramanik, S. (2014), Multi-criteria Group Decision Making Approach for Teacher Recruitment in Higher Education under Simplified Neutrosophic Environment. Neutrosophic Sets and Systems, 6, 28-34; [10] Pouresmaeil, H., Shivanian, E., Khorram, E., Fathabadi, H.S. (2017), An Extended Method Using TOPSIS and VIKOR for Multiple Attribute Decision Making with Multiple Decision Makers and Single Valued Neutrosophic Numbers. Advanced Applied Statistic, 50, 261-292;

[11] Pramanik, S., Dey, P.P., Giri, B.C. (2015), TOPSIS for Single Valued Neutrosophic Soft Expert Set Based Multi-Attribute Decision Making Problems. Neutrosophic Sets and Systems, 10, 88-95;

[12] Sahin, R. (2014), Multi-criteria Neutrosophic Decision Making Method Based on Score and Accuracy Functions under Neutrosophic Environment. arXiv preprint arXiv:1412.5202;

DOI: 10.24818/18423264/55.2.21.01 
Dragisa Stanujkic, Darjan Karabasevic, Gabrijela Popovic, Florentin Smarandache, Edmundas Kazimieras Zavadskas, Ieva Meidutè-Kavaliauskienė

[13] Salton, G., McGill, M.J. (1983), Introduction to Modern Information Retrieval. McGraw-Hill, Auckland;

[14] Smarandache, F. (1999), A Unifying Field in Logics. Neutrosophy:

Neutrosophic Probability, Set and Logic. American Research Press, Rehoboth;

[15] Stanujkic, D., Karabasevic, D., Smarandache, F., Zavadskas, E.K., Maksimovic, M. (2019), An Innovative Approach to Evaluation of the Quality of Websites in the Tourism Industry: a Novel MCDM Approach Based on Bipolar Neutrosophic Numbers and the Hamming Distance. Transformations in Business and Economics, 18(1): 149-162;

[16] Stanujkic, D., Karabasevic, D., Zavadskas, E. K. (2017), A New Approach for Selecting Alternatives Based on the Adapted Weighted Sum and the SWARA Methods: A Case of Personnel Selection. Economic Computation \& Economic Cybernetics Studies \& Research, 51(3): 39-56;ASE Publishing, Bucharest;

[17] Turksen, I.B. (1986), Interval Valued Fuzzy Sets Based on Normal Forms. Fuzzy Sets and Systems, 20(2): 191-210;

[18] Ulutaş, A., Popović, G., Stanujkić, D., Karabašević, D., Zavadskas, E.K., Turskis, Z. (2020), A New Hybrid MCDM Model for Personnel Selection Based on a Novel Grey PIPRECIA and Grey OCRA Methods. Mathematics, 8(10): 1698;

[19] Wang, H., Smarandache, F., Zhang, Y., Sunderraman, R. (2010), Single Valued Neutrosophic Sets. Review of the Air Force Academy, 1(16): 10-14; [20] Yager, R.R. (2013), Pythagorean Fuzzy Subsets. In Proc. Joint IFSA World Congress and NAFIPS Annual Meeting, Edmonton, Canada, pp. 57-61;

[21] Ye, J. (2014), Vector Similarity Measures of Simplified Neutrosophic Sets and Their Application in Multicriteria Decision Making. International Journal of Fuzzy Systems, 16(2): 204-211;

[22] Ye, J. (2015), An Extended TOPSIS Method for Multiple Attribute Group Decision Making Based on Single Valued Neutrosophic Linguistic Numbers. Journal of Intelligent \& Fuzzy Systems, 28(1): 247-255;

[23] Ye, J. (2017), Single-Valued Neutrosophic Similarity Measures Based on Cotangent Function and Their Application in the Fault Diagnosis of Steam Turbine. Soft Computing, 21(3): 817-825;

[24] Ye, J., Zhang, Q.S. (2014), Single Valued Neutrosophic Similarity Measures for Multiple Attribute Decision Making. Neutrosophic Sets and Systems, 2, 48-54;

[25] Zadeh, L.A. (1965), Fuzzy Sets. Information and Control, 8(3): 338-353. 